\title{
A decrease in the expression of CD63 tetraspanin protein elevates invasive potential of human melanoma cells
}

\author{
Hwa-In Jang ${ }^{1}$ and Hansoo Lee Le, $^{1,2}$ \\ ${ }^{1}$ Vascular System Research Center \\ Division of Life Sciences \\ Kangwon National University \\ Chunchon 200-701, Korea \\ ${ }^{2}$ Corresponding author: Tel, 82-33-250-8530; \\ Fax, 82-33-251-3990; E-mail, hslee@kangwon.ac.kr
}

Accepted 22 July 2003

Abbreviations: ECM, extracellular matrix; MMP, matrix metalloproteinase; TM4SF, transmembrane 4 superfamily

\begin{abstract}
CD63, which belongs to the tetraspanin membrane proteins, has been proposed to play an important role in inhibiting melanoma metastasis. To determine whether reduction of CD63 expression, which frequently occurs in the malignant progression of human melanoma, is responsible for metastasis promotion, we transfected the antisense CD63 cDNA into MelJuso melanoma cells having endogenous CD63 expression. The antisense CD63 transfectant clones showing decreased CD63 expression displayed increased cell motility, matrixdegrading activity, and invasiveness in vitro when compared with the control transfectant cells. The antisense CD63 cDNA-transfected cells also exhibited altered adhesiveness to extracellular matrix. The results suggest that reduced CD63 expression contributes to the invasive and metastatic ability of human melanoma cells.
\end{abstract}

Keywords: cancer invasion; CD63; cell motility; melanoma; metalloproteinase

\section{Introduction}

Tetraspanins, or transmembrane 4 superfamily (TM4SF) molecules, are characterized by the existence of four highly conserved transmembrane domains and some members of this family such as CD9, CD63, CD81, CD82, and CD151 are ubiquitously expressed whereas others are highly restricted to immune cells (Maecker et al., 1997; Boucheix and Rubinstein, 2001;
Yanez-Mo et al., 2001, for reviews). The tetraspanin molecules have been implicated in diverse biological phenomena, including cell proliferation, activation, adhesion, migration, differentiation, and development. Some of these molecules have also been implicated in cancer metastasis: CD9 expression is inversely correlated with the appearance of metastases in melanomas, breast, lung, and colon cancers (Si and Hersey, 1993; Miyake et al., 1995; Adachi et al., 1998; Mori et al., 1998). CD82, which was rediscovered as the KAl1 metastasis-suppressor gene product, also become down-regulated in the malignant progression of many types of human cancers, including prostate, breast, pancreatic, lung, bladder, and gastric cancer (Guo et al., 1996; Ueda et al., 1996; Yang et al., 1997; Yu et al., 1997; Higashiyama et al., 1998; Hinoda et al., 1998). Several transfection studies have demonstrated the metastasis-suppressing role of CD9 and CD82 in cancer cells (Ikeyama et al., 1993; Dong et al., 1995; Takaoka et al., 1998a; 1998b; Miyake et al., 2000; Yang et al., 2001).

CD63 tetraspanin protein, which has also been identified as a melanoma-associated antigen ME491, is another candidate that might play a role in cancer metastasis. CD63 is expressed in several normal tissues as well as in most cultured melanoma cell lines (Sikora et al., 1987; Metzelaar et al., 1991). Interestingly, CD63 is strongly expressed in early stages of melanoma, but its expression is weaker or absent in late malignant stages, suggesting some role of CD63 in malignant progression of melanoma (Atkinson et al., 1984; Kondoh et al., 1993). Transfection of CD63 into a CD63-negative melanoma cell line reduces metastasis in vivo and this effect is related to the suppression of tumourigenicity and cell motility (Radford et al., 1995; 1997). It therefore appeared likely that CD63 expression inhibits metastasis of human melanomas. However, it is not certain at present that CD63 down-regulation which occurs in melanoma progression indeed gives rise to the elevation of metastatic potential of melanoma cells.

In this study, we have addressed whether reduction of CD63 expression is associated with or causes highly metastatic behavior in melanoma cells. We transfected CD63 cDNA in antisense direction into a human melanoma cell line having endogenous CD63 expression, MelJuso, and examined the effect of reduced CD63 expression on the metastatic phenotype of MelJuso melanoma cells in vitro. 


\section{Materials and Methods}

\section{Cell culture}

C8161 and MelJuso human melanoma cell lines were cultured in DMEM/F-12 medium supplemented with $10 \%$ fetal bovine serum (FBS), $100 \mathrm{U} / \mathrm{ml}$ penicillin, and 100 $\mu \mathrm{g} / \mathrm{ml}$ streptomycin (all from Gibco-BRL, Grand Island, NY) in $5 \% \quad \mathrm{CO}_{2}$ at $37^{\circ} \mathrm{C}$.

\section{Reverse transcriptase-polymerase chain reaction (RT-PCR) analysis}

Total cellular RNA was purified from the cultured cells using Trizol reagent (Gibco-BRL) according to the manufacturer's protocol. First strand CDNA synthesis was performed with $1 \mu \mathrm{g}$ of total RNA using a cDNA synthesis kit (Promega, Madison, WI). For PCR amplification, 5'-TAGATTCGGCAGCCATGGCGGTGGAA-3' was used as the sense primer and 5'-ACTGACCAGACCCCTACATCACC-3' as the antisense primer. This primer pair amplifies a 710-bp fragment of CD63 cDNA. The reaction mixture was subjected to 25 PCR amplification cycles of $60 \mathrm{~s}$ at $94^{\circ} \mathrm{C}, 90 \mathrm{~s}$ at $55^{\circ} \mathrm{C}$, and 90 $\mathrm{S}$ at $72^{\circ} \mathrm{C}$. To obtain reproducible performance of the RT-PCR assay for CD63, $\beta$-actin mRNA in total cellular RNA was also analyzed in the same way.

\section{Transfection of antisense CD63 cDNA and selection of stable clones}

Antisense cDNA fragment $(+70$ to +229$)$ of CD63 was subcloned into the EcoRI/Kpnl sites of pcDNA3 vector (Invitrogen, San Diego, CA), downstream of CMV promoter. The antisense CD63 cDNA expression construct was transfected into MelJuso human melanoma cells by using lipofectAMINE (Gibco-BRL) according to the manufacturer's instructions. pcDNA3 vector only was also transfected as a control. Neomycin-resistant clones were isolated by growing the cells in DMEM/F12 containing $10 \%$ FBS and $0.5 \mathrm{mg} / \mathrm{ml} \mathrm{G} 418$ (Gibco$B R L)$. Stable transfectant clones were characterized by flow-cytometric analysis for their expressions of CD63 protein on cell surface.

\section{Flow-cytometric analysis}

Cells were incubated with $10 \mu \mathrm{g} / \mathrm{ml}$ anti-CD63 monoclonal antibody H5C6 (PharMingen, San Diego, CA) for $30 \mathrm{~min}$, washed with cold PBS, and then incubated with saturating concentrations of FITC-conjugated goat anti-mouse lgG (PharMingen) for $30 \mathrm{~min}$ at $4^{\circ} \mathrm{C}$. After being washed with PBS, the cells were fixed with $2 \%$ formaldehyde in PBS. Cell surface immunofluorescence was analyzed by flow cytometry performed on a FACScan (Becton Dickinson, San Diego, CA).

\section{In vitro invasion assay}

Cells were tested for their invasive ability through membrane filter thickly coated with basement membrane Matrigel (Becton Dickinson) in vitro in Boyden chambers as described (Albini et al., 1987; Lee and Lee, 1999). The lower compartment of 24-well Transwell chambers (Corning Costar, Cambridge, MA) was filled with $0.7 \mathrm{ml}$ of NIH3T3 culture supernatant and the upper chamber was filled with $0.2 \mathrm{ml}$ of the transfectant cell suspension $\left(2 \cdot 10^{5}\right.$ cells $\left./ \mathrm{ml}\right)$. After $24 \mathrm{~h}$ incubation in $5 \% \mathrm{CO}_{2}$ at $37^{\circ} \mathrm{C}$, cells that had passed through the membrane filter and adhered to the lower chamber were stained with hematoxylin and eosin reagent and counted under a microscope.

\section{Migration assay}

Cells $\left(5 \cdot 10^{5}\right)$ were seeded in a well of 24 -well culture plate. When the cells reached a confluent state, the cell layer was wounded with a plastic micropipette tip having large orifice. The medium and debris were aspirated away and replaced by $2 \mathrm{ml}$ of fresh medium. Photographs of wounded area were taken every $24 \mathrm{~h}$ after wound was made, and cell motility was quantified by measuring the migration distance of each group of cells.

\section{Zymogram assay}

Gelatinolytic activities present in conditioned medium were visualized by electrophoresis on SDS-substrate gels as described (Hibbs et al., 1985). Culture medium from $5 \cdot 10^{5}$ cells was mixed $3: 1$ with substrate gel sample buffer $(40 \%$ (v/v) glycerol, 0.25 M Tris$\mathrm{HCl}, \mathrm{pH} 6.8$ and $0.1 \%$ bromophenol blue) and loaded without boiling onto $10 \%$ SDS-polyacrylamide gel containing type I gelatin $(1.5 \mathrm{mg} / \mathrm{ml})$. After electrophoresis at $4^{\circ} \mathrm{C}$, the gel was soaked in $2.5 \%$ Triton X-100 with gentle shaking for $30 \mathrm{~min}$ with one change of detergent solution. The gel was rinsed and incubated overnight at $37^{\circ} \mathrm{C}$ in substrate buffer $(50 \mathrm{mM}$ Tris- $\mathrm{HCl}$, $\mathrm{pH} 7.5,5 \mathrm{mM} \mathrm{CaCl}_{2}$ and $0.02 \% \mathrm{NaN}_{3}$ ). Following the incubation, the gel was stained with $0.05 \%$ Coomassie brilliant blue G-250 and destained in 10\% acetic acid and $20 \%$ methanol.

\section{Adhesion assay}

Twenty four-well tissue culture plates were coated with Matrigel, collagen type IV, fibronectin, or laminin at a concentration of $10 \mu \mathrm{g} / \mathrm{cm}^{2}$ for $1 \mathrm{~h}$ at room temperature. Cells $\left(5 \cdot 10^{4}\right)$ suspended in DMEM/F-12 medium containing $0.5 \%(\mathrm{w} / \mathrm{v}) \mathrm{BSA}$ were dispensed into each well of 24-well culture plate, incubated in $5 \% \quad \mathrm{CO}_{2}$ at $37^{\circ} \mathrm{C}$ for $1 \mathrm{~h}$ and gently washed three times with PBS. Cells attached to the bottom of plate 
A

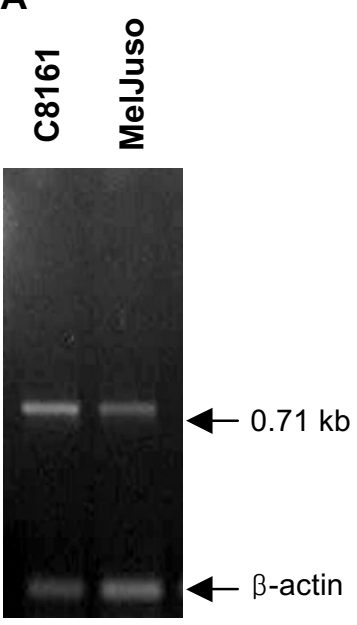

B

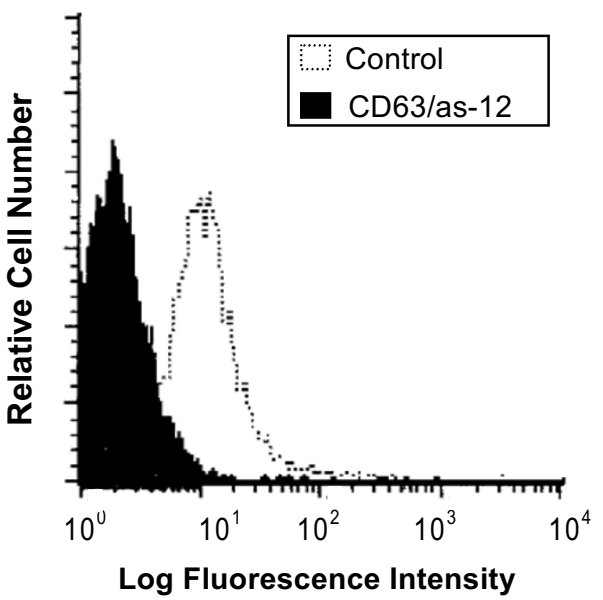

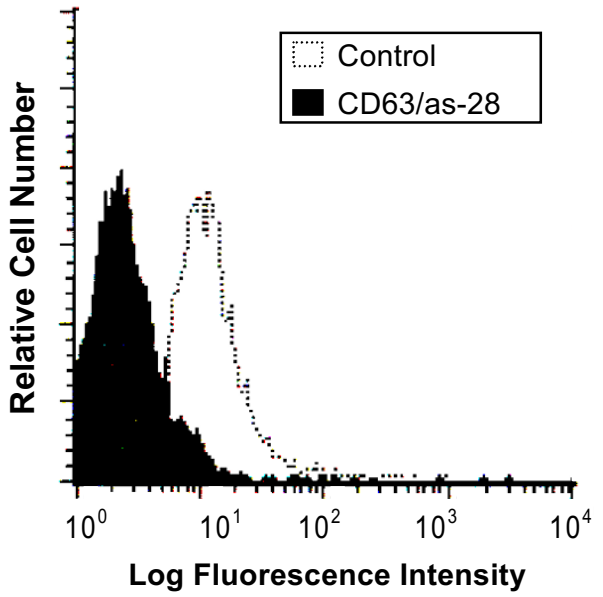

Figure 1. CD63 expression in the parental lines and transfectant clones of human melanoma cells. (A) CD63 mRNAs in total RNA (1 $\mu \mathrm{g}$ ) obtained from two human melanoma cell lines, C8161 and MelJuso, were amplified by RT-PCR, and then electrophoresed on a $1.5 \%$ agarose gel. $\beta$-actin mRNA from each cell line was also analyzed to control for equal RNA amounts. (B) CD63 protein in antisense CD63 cDNA-transfected MelJuso stable clones were analyzed by flow cytometry using anti-CD63 monoclonal antibody. Relative expression of CD63 protein between the antisense CD63 transfectant clones and the control transfectant is shown.

were stained with hematoxylin and eosin reagent and counted under a microscope.

\section{Results}

We performed RT-PCR analysis using primers specific for CD63 cDNA to examine the expression of CD63 message in two human melanoma cell lines, C8161 and MelJuso. A 710-bp PCR product was detected in both melanoma cell lines, indicating that both C8161 and MelJuso are CD63-positive cell lines (Figure 1A). To determine whether the metastatic potential of melanoma cells is indeed elevated by CD63 down-regulation that frequently occurs in the malignant progression of melanomas, MelJuso cells with endogenous CD63 expression were transfected with antisense CD63 cDNA. Expression of CD63 protein in resultant transfectant clones was analyzed by flow cytometry using CD63 monoclonal antibody (Figure 1B). Several transfectant clones obtained with the antisense CD63 cDNA expression vector displayed decreased levels of CD63 protein on cell surface when compared with parental MelJuso cells. Two of these clones, CD63/as-12 and CD63/as-28, which exhibit much lower levels of CD63 protein than the control transfectant generated with an empty vector, were selected for further functional analyses.

To investigate the functional effect of the reduced CD63 expression on cellular functions related to cancer metastasis process, the invasive efficacy of each transfectant in vitro was first determined in Boyden

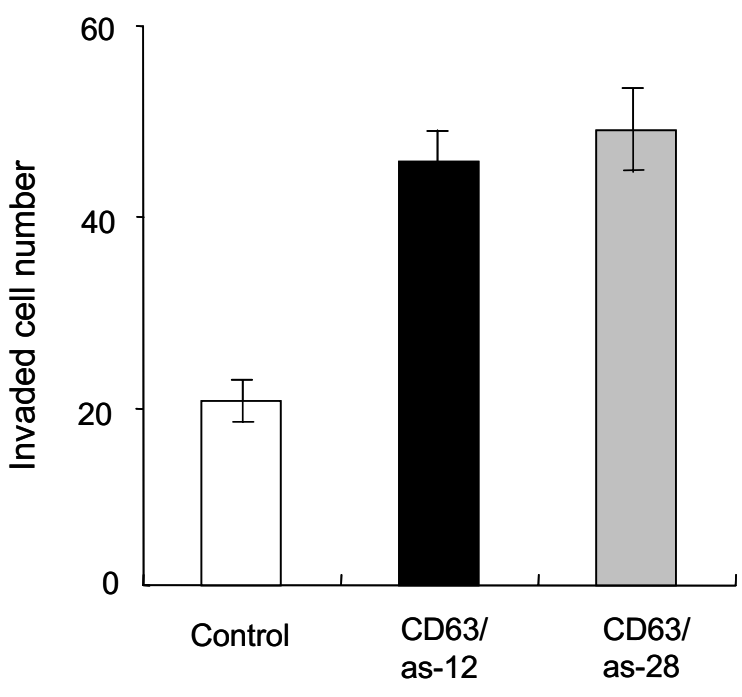

Figure 2. In vitro invasiveness of the antisense CD63 CDNAtransfected MelJuso clones. Each transfectant clone $\left(4 \times 10^{4}\right.$ cells $)$ was seeded in a Transwell chamber insert equipped with reconstituted basement membrane-coated filter. After $24 \mathrm{~h}$ of incubation, cells that had passed through the filter and adhered to the lower chamber were stained with hematoxylin and eosin reagent and counted under a microscope. Data represent the mean \pm SEM of triplicate cultures. The difference in invasiveness between the control and antisense CD63 transfectant cells was statistically significant $(P<0.01$, Student's $t$-test).

chamber assay using basement membrane Matrigel as a cell-migrating barrier. Figure 2 shows comparative invasiveness of the control vector- and antisense CD63 cDNA-transfected MelJuso melanoma cells over 


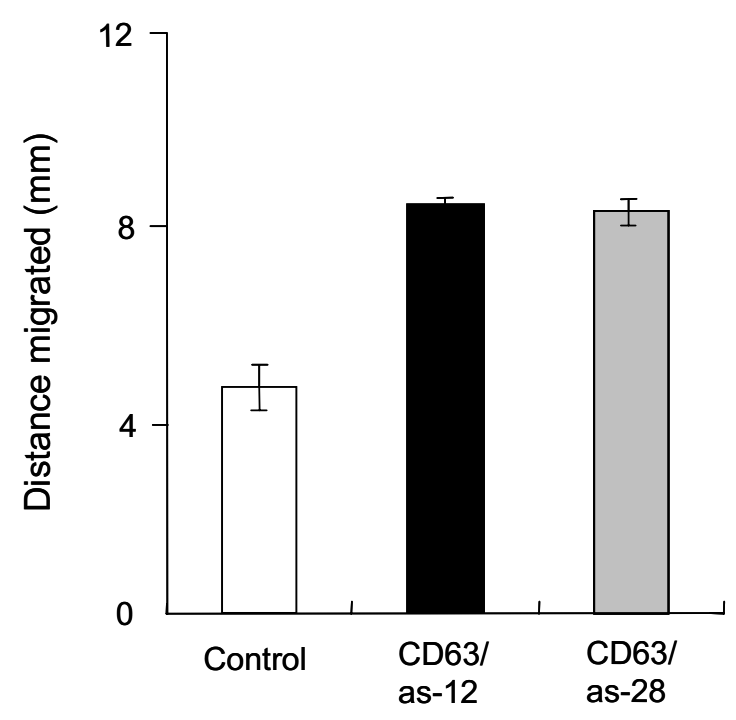

Figure 3. In vitro cell motility of the antisense CD63 transfectant clones. Confluent cultures of the transfectant clones were wounded with a plastic micropipette tip. Photographs were taken at $48 \mathrm{~h}$ after wound was made, and the migration distance of each cell was measured for cell motility. Shown in the figure are means \pm SEMs $(n>30)$. The difference in motility between the control and antisense CD63 transfectant cells was statistically significant $(P<0.01$, Student's $t$-test).

1

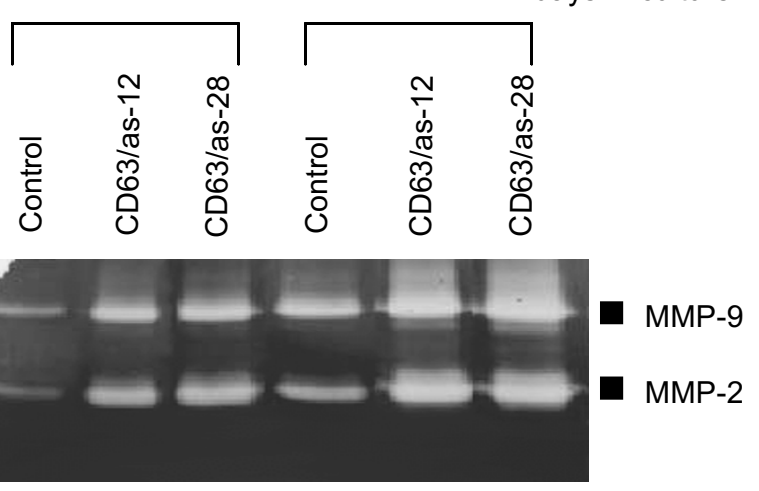

Figure 4. Zymogram depicting MMP-2/-9 activity in conditioned media of the antisense CD63 transfectant clones. Conditioned medium obtained from the culture of each transfectant clone was electrophoresed on a $10 \%$ SDS-polyacrylamide gel. After removal of SDS, the gel was incubated in gelatinase substrate buffer and then visualized by Coomassie staining.

$24 \mathrm{~h}$ of culture by use of fibroblast culture supernatant as a chemoattractant. The antisense CD63 transfectant clones showed a 2-fold higher invasiveness than the control transfectant cells. The result in in vitro invasion assay strongly suggests that the decrease in CD63 expression promotes invasion process of melanoma.

As an effort to reveal what kinds of cellular characteristics involved in cancer invasion process are reg-

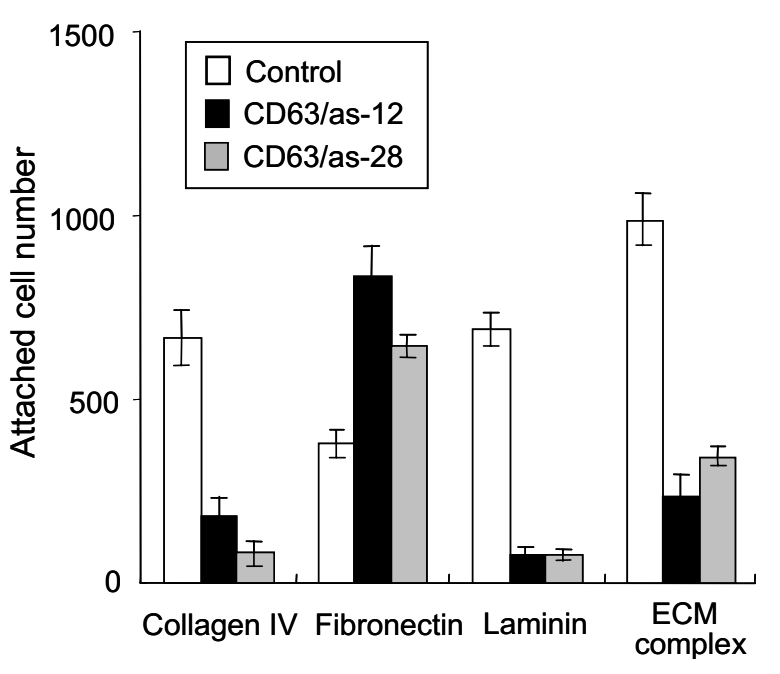

Figure 5. Adhesion of the antisense CD63 transfectant clones to extracellular matrix proteins. Each transfectant clone $\left(5 \times 10^{4}\right.$ cells $)$ was dispensed into each well of 24-well plate that had been coated with Matrigel, collagen type IV, fibronectin, or laminin at a concentration of $10 \mu \mathrm{g} / \mathrm{cm}^{2}$, respectively. After $1 \mathrm{~h}$ of incubation, unbound cells were aspirated, and adherent cells were stained with hematoxylin and eosin reagent and counted under a microscope. Data represent the mean \pm SEM of triplicate determinations in two independent experiments. The difference in adhesion to collagen type IV, fibronectin, laminin, and Matrigel between the control and antisense CD63 transfectant clones was statistically significant $(P<0.01$, Student's t-test).

ulated by the decrease in CD63 expression, we examined the migrating ability of the antisense CD63 CDNAtransfected MelJuso cells into a wounded space on culture plate. Both transfectant clones, CD63/as-12 and CD63/as-28, exhibited a 1.5 -fold higher migrating ability than the control transfectant (Figure 3). It thus appears that the decrease in CD63 expression enhances the motility of melanoma cells. The basement membrane-degrading ability of the transfectant clones was also examined by measuring gelatinase activity in culture supernatant. As a result, two types of matrix metalloproteinase (MMP) exhibiting collagen-degrading activity, MMP-2 of $72 \mathrm{kDa}$ size and MMP-9 of $92 \mathrm{kDa}$ size, were detected in the culture supernatant of MelJuso cells (Figure 4). The activity of both MMP enzymes in the antisense CD63 transfectant clones was much higher than that in the control transfectant, indicating that reduced CD63 expression enhances surrounding matrix-degrading activity of MelJuso melanoma cells. Therefore, it seems likely that the invasiveness induced by the decrease in CD63 expression is due to its positive effect on motility and matrixdegrading ability of melanoma cells.

Since alterations in adhesion of cancer cells to extracellular matrix (ECM) components frequently occurs during the process of invasion and metastasis, we examined the effect of reduced CD63 expression 
on the binding of MelJuso cells to ECM components, collagen type IV, fibronectin, and laminin as well as ECM complex, Matrigel. As a result, the antisense CD63 transfectant clones exhibited significantly decreased binding to collagen IV, laminin, and Matrigel in compared to the control transfectant (Figure 5). On the other hand, adhesiveness to fibronectin in the antisense CD63 transfectant clones was found to be much higher than that in the control transfectant. The results in the cell-ECM binding assay indicate that the binding ability of melanoma cells to extracellular matrix can be modulated by CD63 expression.

\section{Discussion}

Previous reports have suggested that CD63 membrane protein may play an important role in inhibiting human melanoma progression. CD63 expression in melanomas is strong in early stage, but weaker or absent in late stage (Atkinson et al., 1984; Kondoh et al., 1993). Overexpression of CD63 was shown to suppress tumorigenicity of $\mathrm{H}$-ras-transformed NIH3T3 cells in athymic nude mouse (Hotta et al., 1991). Transfection of genomic CD63 into a CD63-negative melanoma cell line did not only inhibit growth in vivo, but also reduced metastatic potential of the melanoma cells (Radford et al., 1995). Motility of the CD63-negetive melanoma cells was also suppressed by CD63 transfection (Radford et al., 1997). In addition, the CD63-transfected melanoma cells were found to be more adhesive to ECM proteins such as collagen, laminin, and fibronectin after the induction of CD63 expression (Radford et al., 1997). It thus appears that CD63 expression induced by transfection in CD63negative cells suppresses metastatic ability of the melanoma cells.

In the current report, we used a different approach to demonstrate the metastasis-suppressing role of CD63 in melanoma; that is, a CD63-positve melanoma cell line, MelJuso was transfected with antisense CD63 cDNA to decrease the expression level of CD63, and in vitro invasiveness of the resultant transfectant clones was examined. The decrease in CD63 expression by transfection with antisense CD63 cDNA may reflect a situation of CD63 down-regulation that actually occurs in the malignant progression of human melanoma. Our finding that the decease in CD63 expression enhanced the invasive ability of MelJuso melanoma cells strongly support the idea that reduced CD63 expression contributes to development of malignant phenotypes such as invasion and metastasis.

Tumor cell invasion involves the following closely related processes: tumor cell separation from the primary mass, proteolysis of the surrounding stroma and extracellular matrix (ECM), and cell migration into spaces cleared by proteolysis followed by penetration through endothelial cells. In this study, we have found that reduced CD63 expression in MelJuso cells results in the increases in cell motility and activity of matrix-degrading enzymes such as MMP-2/-9 (Figure 3 and 4). Also, the antisense CD63 cDNA-transfected cells displayed decreased binding ability to ECM proteins such as collagen and laminin as well as ECM complex, Matrigel, when compared to the control transfectant cells (Figure 5). Although no effect of CD63 expression was observed on homotypic adhesion of MelJuso cells (data not shown), the decreased cell adhesion to ECM would allow melanoma cell separation from the primary tumor mass. Therefore, enhancement of invasive potential of melanoma cells by the decrease in CD63 expression might be due to the positive effect of reduced CD63 expression on cell motility, matrix proteolytic capability, and ability to detach from the surrounding matrix.

CD63 has been shown to associate with other tetraspanin proteins, CD9 and CD81, and with $\beta_{1}$ integrins in human melanoma cells (Radford et al., 1996). Among these molecules, CD9 plays a role in inhibiting melanoma metastasis since its expression induced by transfection or viral delivery of CD9 gene was found to suppress motility and metastasis of mouse melanoma cells (Ikeyama et al., 1993; Miyake et al., 2000). CD9 expression has also been inversely related to metastatic potential of melanoma ( $\mathrm{Si}$ and Hersey, 1993). It thus appears that melanoma metastasis is not inhibited only by CD63, but also by CD9. In addition, integrins have been known to play an important role in regulating the invasive and metastatic ability of tumor cells. Especially, it was reported that $\alpha_{3} \quad \beta_{1}$ integrin is involved in the invasiveness of melanoma, along with tumorigenicity and cell motility (Natali et al., 1993; Melchiori et al., 1995). Therefore, the effect of CD63 expression on melanoma metastasis may partly reflect the engagement of associated molecules, including CD9, CD81, and $\beta_{1}$ integrins. To determine whether the effect of CD63 is directly on metastasis, or indirectly through associated molecules with it, the functional significance of the association between $\operatorname{CD} 63$ and $\beta_{1}$ integrins, and other tetraspanin proteins such as CD9, CD81 in melanoma metastasis needs to be clarified.

\section{Acknowledgement}

This work was supported by Vascular System Research Grant from the Korean Science and Engineering Foundation. 


\section{References}

Adachi M, Taki T, Konishi T, Huang $\mathrm{Cl}$, Higashiyama M, Miyake M. Novel staging protocol for non-small-cell lung cancers according to MRP-1/CD9 and KAl1/CD82 gene expression. J Clin Oncol 1998;16:1397-406

Albini A, Iwamoto $Y$, Kleinman HK, Martin GR, Aaronson SA, Kozlowski JM, McEwan RM. Rapid in vitro assay for quantitating the invasive potential of tumor cells. Cancer Res 1987; $47: 3239-45$

Atkinson B, Ernst CS, Ghrist BF, Herlyn M, Blaszczyk M, Ross AH, Herlyn D, Steplewski Z, Koprowski H. Identification of melanoma-associated antigens using fixed tissue screening of antibodies. Cancer Res 1984;44:2577-81

Boucheix C, Rubinstein E. Tetraspanins. Cell Mol Life Sci 2001;58:1189-205

Dong JT, Lamb PW, Rinker-Schaeffer CW, Vukanovic J, Ichikawa T, Isaacs JT, Barrett JC. KAl1, a metastasis suppressor gene for prostate cancer on human chromosome 11p11.2. Science 1995;268:884-6

Guo X, Freiss H, Graber HU, Kashiwagi M, Zimmermann A, Korc M, Buchler MW. KAI1 expression is up-regulated in early pancreatic cancer and decreased in the presence of metastases. Cancer Res 1996;56:4876-80

Hibbs MS, Hasty KA, Seyer JM, Kang AH, Mainardi CL. Biochemical and immunological characterization of the secreted forms of human neutrophil gelatinase. J Biol Chem $1985 ; 260: 2493-500$

Higashiyama M, Kodama K, Yokouchi H, Takami K, Adachi M, Taki T, Ishiguro S, Nakamori S, Yoshie O, Miyake M. KAI1/CD82 expression in nonsmall cell lung carcinoma is a novel, favorable prognostic factor: an immunohistochemical analysis. Cancer 1998;83:466-74

Hinoda $Y$, Adachi $Y$, Takaoka A, Mitsuuchi H, Satoh $Y$, Itoh $F$, Kondoh $Y$, Imai K. Decreased expression of the metastasis suppressor gene KAl1 in gastric cancer. Cancer Lett 1998;129:229-34

Hotta $\mathrm{H}$, Hara I, Miyamoto $\mathrm{H}$, Homma M. Overexpression of the human melanoma-associated antigen ME491 partially suppresses in vivo malignant phenotypes of $\mathrm{H}$-ras-transformed NIH3T3 cell in athymic nude mice. Melanoma Res 1991; 1:125-32

Ikeyama S, Koyama M, Yamaoko M, Sasada R, Miyake M. Suppression of cell motility and metastasis by transfection with human motility-related protein (MRP-1/CD9) DNA. J Exp Med 1993;177:1231-7

Kondoh M, Ueda M, Ichihashi M, Mishima Y. Decreased expression of human melanoma-associated antigen ME491 along the progression of melanoma pre-canceroses to invasive and metastatic melanomas. Melanoma Res 1993;3: $241-5$

Lee HY, Lee $H$. Inhibitory activity of nm23-H1 on invasion and colonization of human prostate carcinoma cells is not mediated by its NDP kinase activity. Cancer Lett 1999;145: 93-9

Maecker HT, Todd SC, Levy S, The tetraspanin superfamily: molecular facilitators. FASEB J 1997:11:428-42

Melchiori A, Mortarini R, Carlone S, Marchisio PC, Anichini A. Noonan DM, Albini A. The $\alpha 3 \beta 1$ integrin is involved in melanoma cell migration and invasion. Exp Cell Res 1995; 219:233-42

Metzelaar MJ, Wijngaard PL, Peters PJ, Sixma JJ, Nieuwenhuis HK, Clevers HC. CD63 antigen. A novel lysosomal membrane glycoprotein, cloned by a screening procedure for intracellular antigens in eukaryotic cells. J Biol Chem 1991; 266:3239-45

Miyake M, Nakano $\mathrm{K}$, Ikei $\mathrm{Y}$, Adachi M, Huang $\mathrm{CL}$, Itoi $\mathrm{S}$, Koh T, Taki T. Motility related protein 1 (MRP-1/CD9) expression: inverse correlation with metastases in breast cancer. Cancer Res 1995;55:4127-31

Miyake $M$, Inufusa $H$, Adachi $M$, Ishida $H$, Hashida $H$, Tokuhara T, Kakehi Y. Suppression of pulmonary metastasis using adenovirally motility related protein-1 (MRP-1/CD9) gene delivery. Oncogene 2000;19:5221-6

Mori M, Mimori K, Shiraishi T, Haraguchi M, Ueo H, Barnard GF, Akiyoshi T. Motility related protein 1 (MRP1/CD9) expression in colon cancer. Clin Cancer Res 1998;4:1507-10

Natali PG, Nicotra MR, Bartolazzi A, Cavaliere R, Bigotti A. Integrin expression in cutaneous malignant melanoma: association of the $\alpha 3 / \beta 1$ heterodimer with tumor progression. Int J Cancer 1993;54:68-72

Radford KJ, Mallesch J, Hersey P. Suppression of human melanoma cell growth and metastasis by the melanomaassociated antigen CD63 (ME491). Int J Cancer 1995;62:631-5

Radford KJ, Thorne RF, Hersey P. CD63 associates with transmembrane 4 superfamily members, CD9 and CD81, and with $\beta 1$ integrins in human melanoma. Biochem Biophys Res Commun 1996;222:13-8

Radford KJ, Thorne RF, Hersey P. Regulation of tumor cell motility and migration by $\mathrm{CD} 63$ in a human melanoma cell line. J Immunol 1997;158:3353-8

Si Z, Hersey P. Expression of the neuroglandular antigen and analogues in melanoma. CD9 expression appears inversely related to metastatic potential of melanoma. Int $\mathrm{J}$ Cancer 1993:54:37-43

Sikora LK, Pinto A, Demetrick DJ, Dixon WT, Urbanski SJ, Temple W, Jerry LM. Characterization of a novel neuroglandular antigen (NGA) expressed on abnormal human melanocytes. Int J Cancer 1987;39:138-45

Takaoka A, Hinoda Y, Sato S, Itoh F, Adachi M, Hareyama $M$, Imai $K$. Reduced invasive and metastatic potentials of KAl1-transfected melanoma cells. Jpn J Cancer Res 1998a; 89:397-404

Takaoka A, Hinoda Y, Sato S, Adachi Y, Itoh F, Adachi M, Imai K. Suppression of invasive properties of colon cancer cells by a metastasis suppressor KAl1 gene. Oncogene 1998b;16:1443-53

Ueda T, Ichikawa T, Tamaru J, Mikata A, Akakura K, Akimoto S, Imai T, Yoshie O, Shiraishi T, Yatani R, Ito $\mathrm{H}$, Shimazaki J. Expression of the KAl1 protein in benign prostatic hyperplasia and prostate cancer. Am J Pathol 1996; 149:1435-40 
Yanez-Mo M, Mittelbrunn M, Sanchez-Madrid F. Tetraspanins and intercellular interactions. Microcirculation 2001;8:153-68

Yang X, Welch DR, Phillips KK, Weissman BE, Wei LL. KAI1, a putative marker for metastatic potential in human breast cancer. Cancer Lett 1997;119:149-55

Yang X, Wei LL, Tang C, Slack R, Mueller S, Lippman ME.
Overexpression of KAl1 suppresses in vitro invasiveness and in vivo metastasis in breast cancer cells. Cancer Res $2001 ; 61: 5284-8$

Yu Y, Yang IL, Markovic B, Jackson P, Yardley G, Barrett $J$, Russell PJ. Loss of KAl1 messenger RNA expression in both high-grade and invasive human bladder cancers. Clin Cancer Res 1997;3:1045-9 